\title{
Development of an EV Drive Torque Control System for Improving Vehicle Handling Performance Through Steering Improvements
}

\author{
Yuuki Shiozawa $^{1}$ Yosuke Kobayashi $^{2}$ Mitsunori Ohta $^{3}$ Toshiyuki Murata $^{4}$ \\ Yusuke Kageyama,Ph.D. ${ }^{5}$ Yohei Nakamura ${ }^{6}$ Hideaki Misawa $^{7}$ \\ ${ }^{I}$ NISSAN MOTOR CO., LTD. \\ 1-1 Morinosatoaoyama,Atsugi-shi, Kanagawa,243-0123, Japan (E-mail: shioz@mail.nissan.co.jp) \\ ${ }^{2 \sim 7}$ NISSAN MOTOR CO., LTD. \\ 1-1 Morinosatoaoyama, Atsugi-shi, Kanagawa, 243-0123, Japan
}

\begin{abstract}
At Nissan we have developed many different environmental technologies to date and launched sales of an electric vehicle (EV) in December 2010 that achieves zero carbon dioxide and zero exhaust emissions. Besides its outstanding environmental performance, this zero-emission EV also embodies new potentialities not found in conventional gasoline-engine vehicles. The responsiveness and controllability of the electrified powertrain (EV drive motor) combine to provide an unprecedented driving experience that transforms conventional thinking about automobiles. This paper presents an EV drive torque control system that takes advantage of this EV-unique performance to provide handling capabilities for driving ease that anyone can enjoy.
\end{abstract}

Keywords: control system, EV (electric vehicle), motor, vehicle performance

\section{Introduction}

At Nissan, we have developed many different technologies over the years for improving the environmental performance of vehicles. In December 2010, we put on the market an electric vehicle (Fig. 1) that achieves zero emissions of carbon dioxide and exhaust pollutants. In developing this EV, emphasis was also placed on producing a highly attractive, fun-to-drive vehicle in addition to achieving outstanding environmental performance. For example, the ideal driving performance of an EV was defined as "nimble smoothness." Three distinct technologies have been used to develop this new EV to provide nimble, smooth driving performance. Specifically; a vehicle mass package with a low center of gravity and a low yaw moment, a high-stiffness body that incorporates the battery case as a structural element, and a drive torque control system that is the subject of this paper.

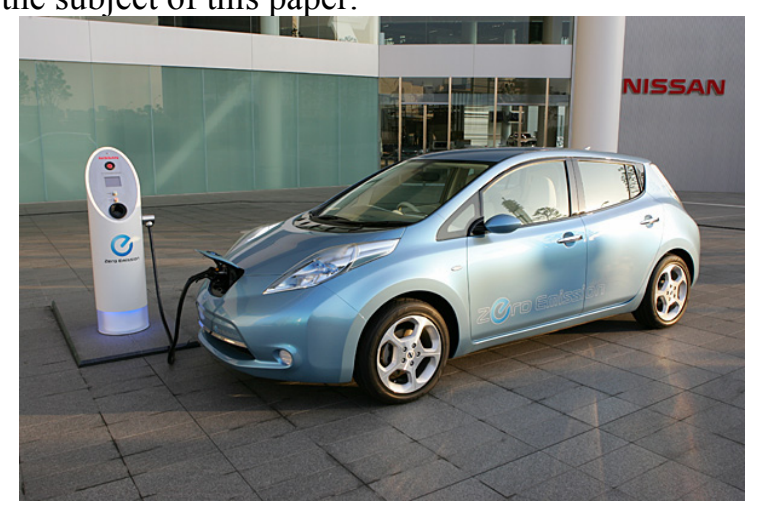

Figure1: Newly developed EV 
This control system was designed with the aim of effectively using the excellent responsiveness and controllability of the EV drive motor to achieve a unique, unprecedented driving experience. This paper describes the measures taken to use the drive torque control system and EV drive motor to obtain handling performance that anyone can enjoy and the specific effects of the methods applied.

\section{Aims of Control System}

\subsection{Index of nimble smoothness}

Comparisons were made between vehicle dynamic data and corresponding subjective evaluation results obtained in various driving situations in order to clarify how a vehicle behaves when the driver perceives nimble, smooth driving performance. The results of this comparative investigation revealed that drivers perceive nimble smoothness when the vehicle responds smoothly and without delay to steering inputs, and also when the vehicle experiences little body lean. This perception was then converted to physical values of vehicle behavior. It was found that two measures in particular are important in achieving the nimble, smooth driving ease that characterizes an EV. One is the improved onset of turning response relative to a steering input, i.e., the initial yaw rate response, and the other is controlled roll rate peak induced during cornering. Ideal vehicle behavior during a steering maneuver is shown in Fig. 2 in terms of time histories of the steering angle, yaw rate and roll rate.

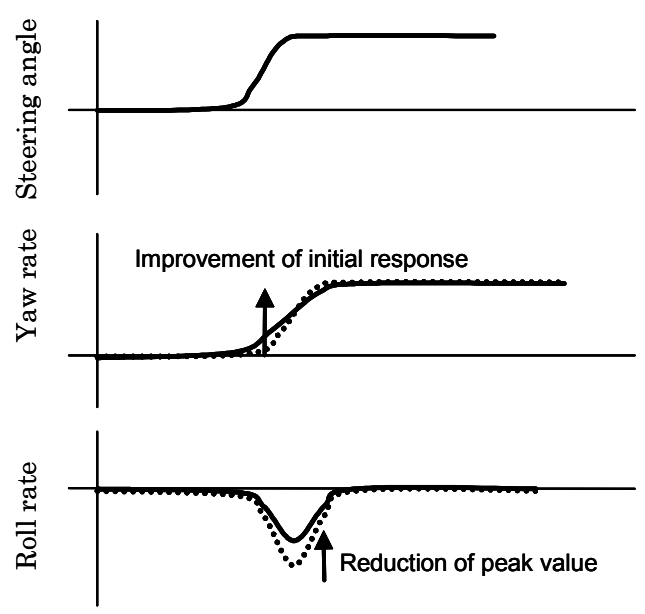

Figure2: Ideal vehicle behavior

\subsection{Relationship between yaw rate and roll rate}

Vehicle roll rate behavior is primarily caused by a centrifugal force (lateral $G$ ) produced during cornering. In everyday driving situations that are the focus of this control system, it is assumed that centrifugal force is nearly proportional to the yaw rate because the vehicle sideslip angular velocity is small. Because of this proportional relationship, a sudden change in the yaw rate means that centrifugal force also changes suddenly, resulting in a larger roll rate. Conversely, the roll rate peak can be controlled if the initial yaw rate response to a steering input can be improved and also if the change in the yaw rate can be moderated without affecting directional control in the latter phase of the turn.

To accomplish that, the control system described here is designed such that the yaw rate response waveform is controlled. Controlling this response waveform makes it possible to simultaneously improve the initial yaw rate response and to suppress the peak roll rate, thereby achieving the aim of improving perceived nimble smoothness.

\section{Mechanism of Using Drive Torque Control to Control Cornering Behavior}

It was shown in the preceding section that the targeted handling performance can be attained by suitably controlling the yaw rate. The drive torque control system proposed here adopts the principle of applying a cornering moment. It also uses the principle of stabilizing the wheel load to obtain the effect of yaw rate control regardless of the driving situation. These two basic principles are explained below.

\subsection{Application of cornering moment}

In a vehicle that is steered and driven by the front wheels, the application of drive torque during cornering generates not only force in the vehicle's longitudinal direction but also a cornering moment. As illustrated in Fig. 3, when drive torque is applied to the outer and inner front wheels while cornering, the right- and left-side moment arms, i.e., the distance from the point acted on by the drive torque to the vehicle's center of gravity, differ depending on the steering angle. Therefore, applying the same magnitude of drive torque to the outer and inner wheels under a cornering condition generates a cornering moment in the vehicle. 


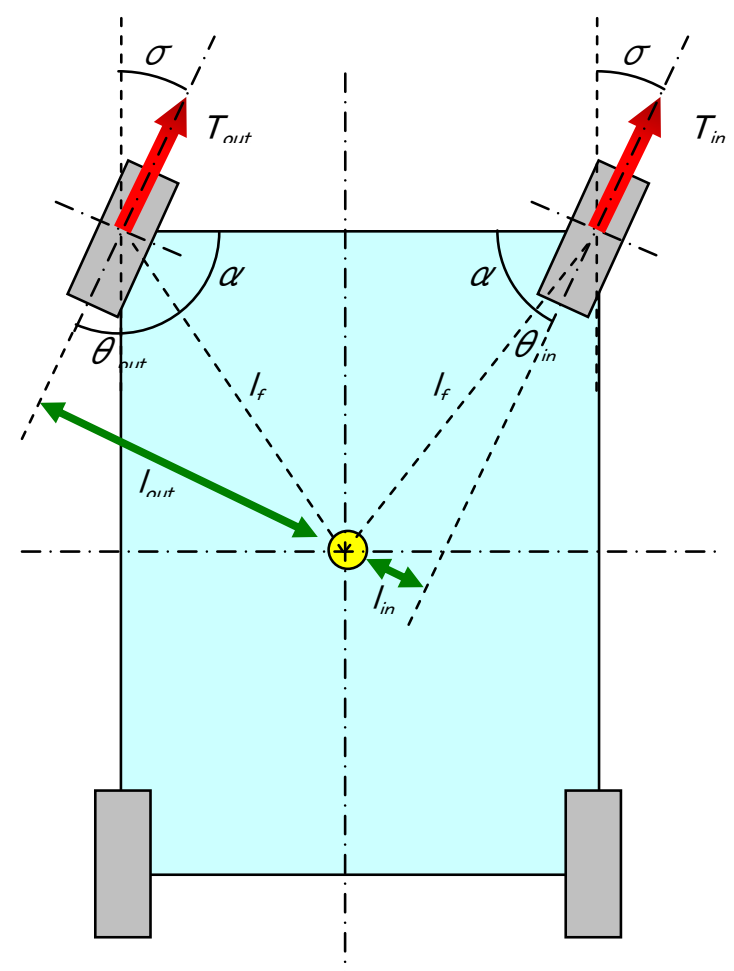

Figure3: Relation between drive torque and cornering moment

In short, increasing the drive torque in a frontsteering, front-drive vehicle while cornering increases the steering moment in the steering direction, whereas reducing the drive torque has the opposite effect of generating a moment in the direction to restrain cornering. This relationship is expressed in the following equation for the cornering moment $\mathrm{M}_{\mathrm{fr}}$.

$$
\begin{aligned}
M_{f r} & =M_{\text {out }}-M_{\text {in }} \\
& =T_{\text {out }} l_{\text {out }}-T_{\text {in }} l_{\text {in }} \\
& =T_{\text {oul }} l_{f} \sin (90+\sigma-\alpha)-T_{\text {in }} l_{f} \sin (90-\sigma-\alpha)
\end{aligned}
$$

\section{Definitions:}

Mfr: cornering moment generated by the front wheels (total for both)

Mout: cornering moment generated by the outer wheel of the turn

Min: cornering moment generated by the inner wheel of the turn

Tout: drive torque at the outer wheel of the turn

Tin: drive torque at the inner wheel of the turn

lout: length of moment arm of outer wheel in the turn to the vehicle' $s$ center of gravity lin: length of moment arm of inner wheel in the turn to the vehicle' $\mathrm{s}$ center of gravity

lf: distance from center of gravity to front wheels

$\alpha: \quad$ angle of elevation of front axle to center of gravity

$\sigma: \quad$ tire steering angle

$\theta$ out: angle of elevation of outer wheel of the turn to center of gravity

$\theta$ in: angle of elevation of inner wheel of the turn to center of gravity

These variables are used to control the yaw rate so as to approach the ideal behavior shown in Fig. 1 by increasing or decreasing the applied drive torque between the first and second phases of a turn. The result of the cornering moment on vehicle behavior is illustrated in Fig. 4.

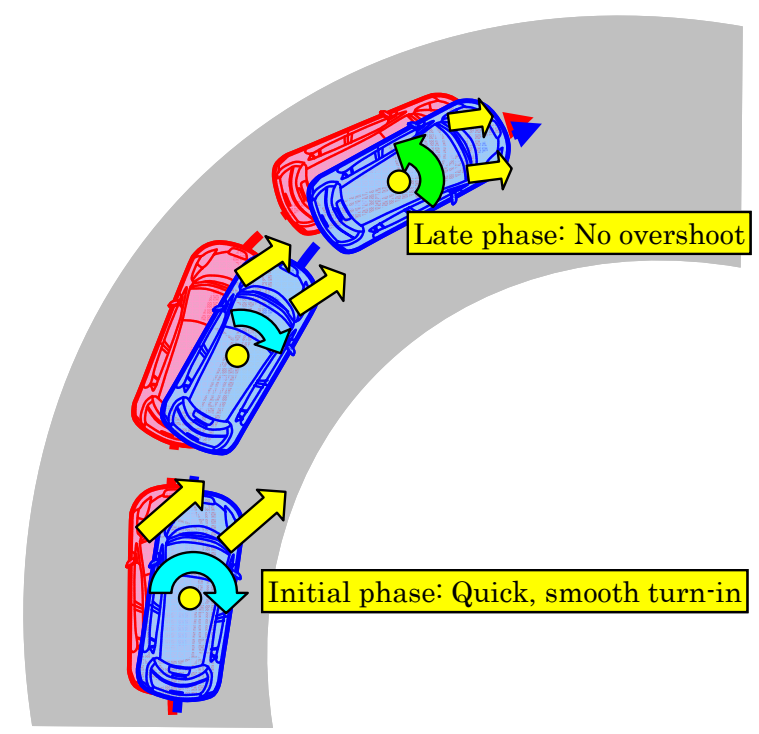

Figure4: Effect of cornering motion on vehicle behavior

\subsection{Stabilization of wheel load}

As described in the preceding subsection, the yaw rate can be changed by increasing or decreasing the drive torque to match the driver's steering input. However, depending on the wheel load, the effect might not be fully obtained because the amount of control applied is either excessive or insufficient. For that reason, it is essential to stabilize the load at each wheel in order to obtain the desired effect regardless of the road surface or driving situation. 

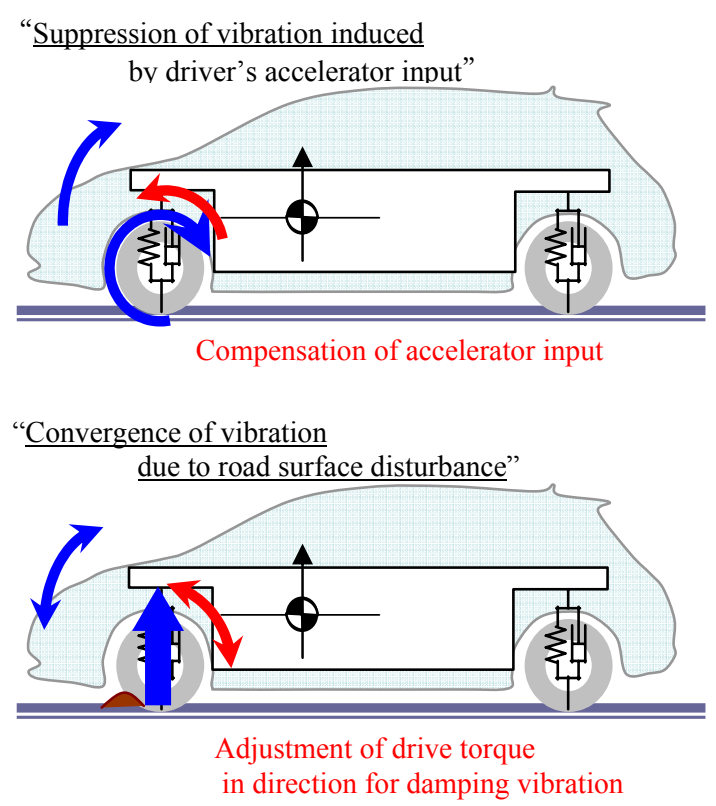

Figure5: Stabilization of wheel load

The pitching and bouncing vibrations of the sprung body induced by the driver's accelerator inputs or road surface disturbances cause the load at each wheel to vary. As illustrated in Fig. 5, these vibrations of the sprung body can be suppressed or damped more quickly by increasing or decreasing the drive torque in the direction that cancels the vibrations, making it possible to stabilize the wheel load.

\section{Overview of Drive Torque Control Logic}

Figure 6 is a block diagram of the control logic that was configured based on the mechanism described in the preceding section.

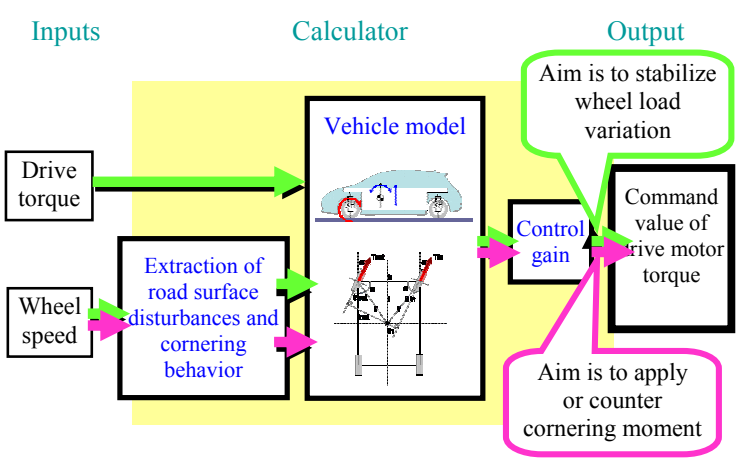

Figure6: Control block diagram

In this control system, the pitching and bouncing behavior of the sprung body is estimated with a vehicle model. The estimate is based on the driver's demanded torque, which is calculated from the driver's accelerator input, and the road surface disturbance which is estimated from the variation in the rotational speed of the four wheels. A command signal value is then calculated for stabilizing the wheel load variation according to the estimated body behavior. In addition, the difference in rotational speed between the front and rear wheels during cornering is translated into a disturbance force and input into the vehicle model. This procedure works to stabilize the wheel load variation while simultaneously applying a cornering moment that is generated by the drive torque to match the driver's steering input.

\section{Effect of Drive Torque Control}

Simulations and driving tests were conducted to validate the basic control principle and confirm the effects of drive torque control. The purpose was to verify whether the mechanism and control logic described above could achieve the nimble smoothness that was defined as the ideal driving performance of an EV.

\subsection{Validation by simulation}

A simulation was run to confirm the change in the cornering moment when drive toque was applied to the front wheels while cornering and a change in the wheel load when drive torque was applied in a direction to cancel body vibration induced by a disturbance, as described in section 3 .

A linear two-wheel model was used to verify the effect of applying a cornering moment. In this model, the cornering moment that is generated around the vehicle's center of gravity due to the applied drive torque is determined from the calculated body sideslip angle and the steering angle as reflected in the vehicle's yaw motion.

Figure 7 shows the simulation results that were obtained when drive torque in the form of a square wave was applied in the acceleration direction in the first half of a turn and in the deceleration direction in the second half.

The results confirm that increasing or decreasing drive torque during cornering improves the initial yaw response and enhances linearity by suppressing overshoot in the second half of the turn. It is clear that positive use of this mechanism can extend the application of drive torque to control cornering behavior, whereas it has so far only been used to control longitudinal vehicle motion. 

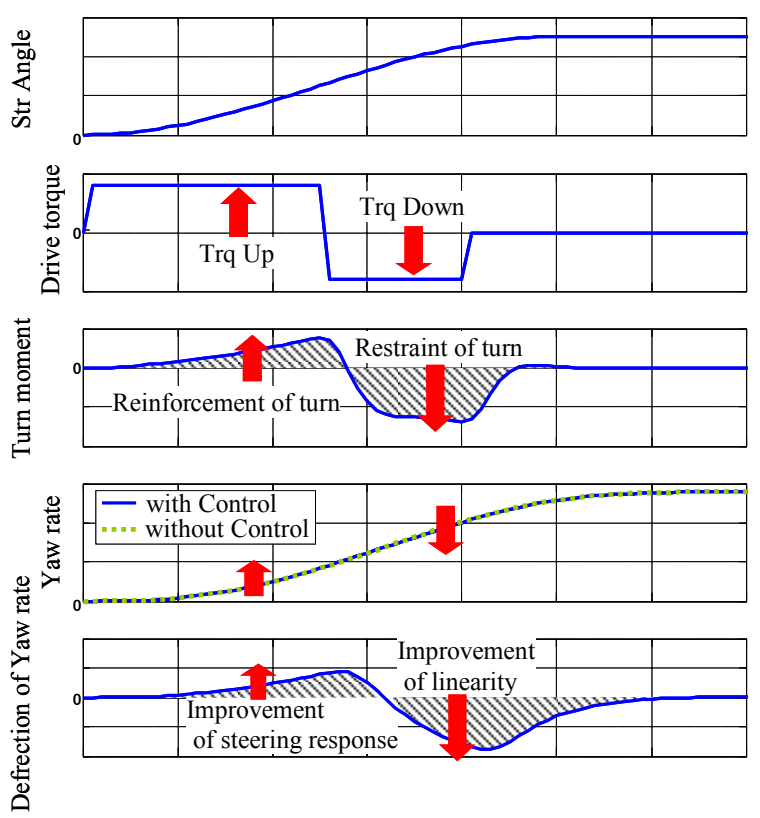

Figure 7: Change in yaw rate due to control system

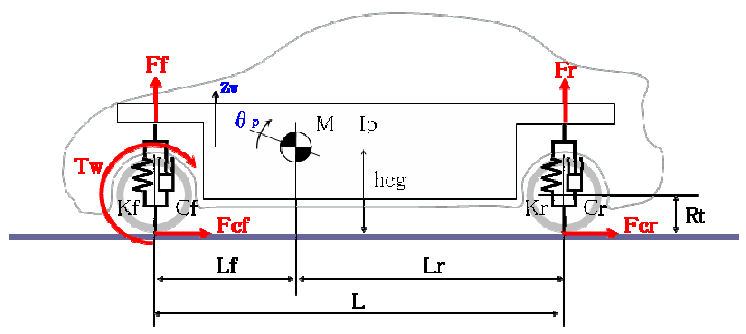

Figure8: Model of sprung body vibration

A simulation was then conducted to verify the effect of applying drive torque on stabilizing the wheel load. This simulation was run using the sprung body vibration model shown in Fig. 8. The symbols in the figure are:

Ff: disturbance force applied to front wheels (total for both)

Fr: disturbance force applied to rear wheels (total for both)

Tw: drive torque

$\mathrm{Zv}$ : body bounce velocity

$\theta$ p: body pitch angular velocity

M: mass of sprung body

Ip: moment of inertia of sprung body (pitch rotation direction)

hcg: height of center of gravity

Lf: distance from center of gravity to front wheels

Lr: distance from center of gravity to rear wheels

L: wheelbase

Rt: dynamic tire radius
Kf: spring coefficient of front wheel suspension (total for both)

Cf: damping coefficient of front wheel shock absorbers (total for both)

$\mathrm{Kr}$ : spring coefficient of rear wheel suspension (total for both)

$\mathrm{Cr}$ : damping coefficient of rear wheel shock absorbers (total for both)

The simulation was conducted to confirm how the wheel load varied with and without the application of drive torque compensation to cancel the disturbance torque input in the pitch rotation direction around the vehicle's center of gravity. The results are shown in Fig. 9.
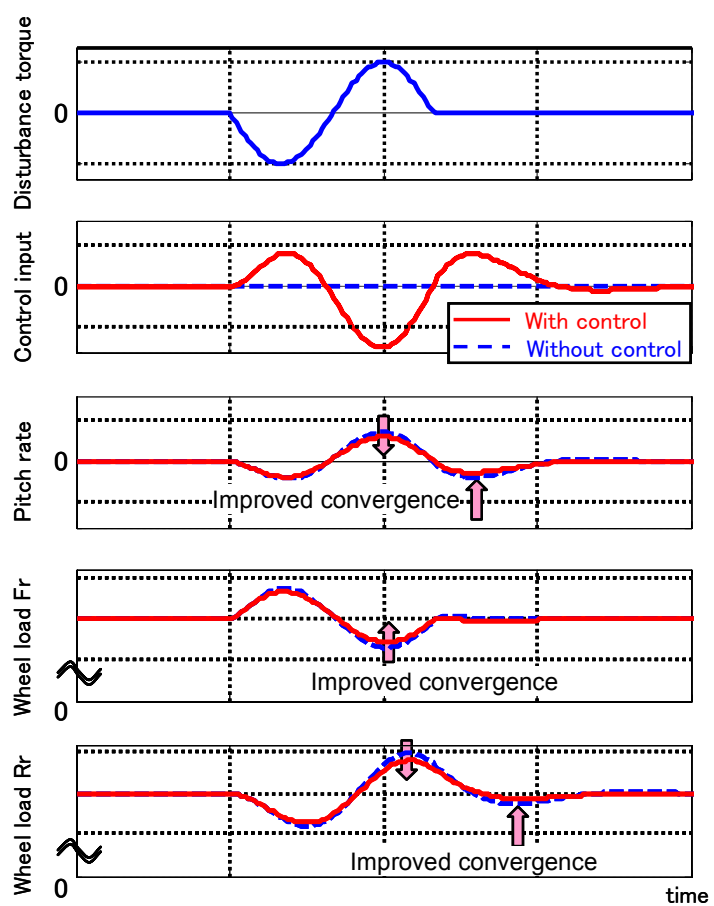

Figure9: Simulation results for stabilization of wheel load

The results indicate that suitably increasing or decreasing the drive torque when vibrations occur in the vehicle body improves the convergence of the vibrations, making it possible to stabilize the wheel load.

\subsection{Validation in driving tests}

The effects of the drive torque control system were confirmed by conducting EV driving tests. Figure 10 shows the test pattern that was used to simulate a lane-change maneuver, and vehicle behavior with and without the control system is compared in Fig. 11. 


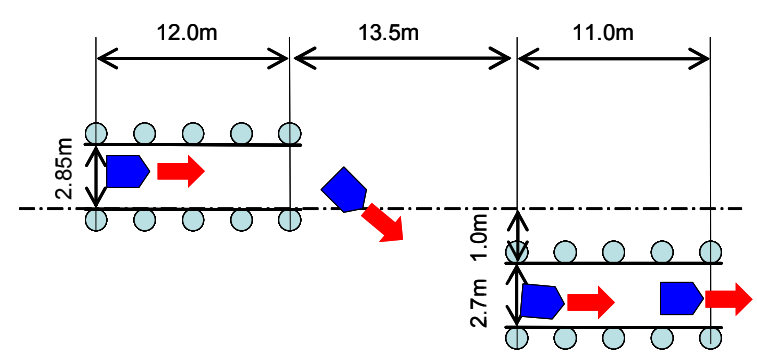

Figure10: Test pattern (Lane-change maneuver)
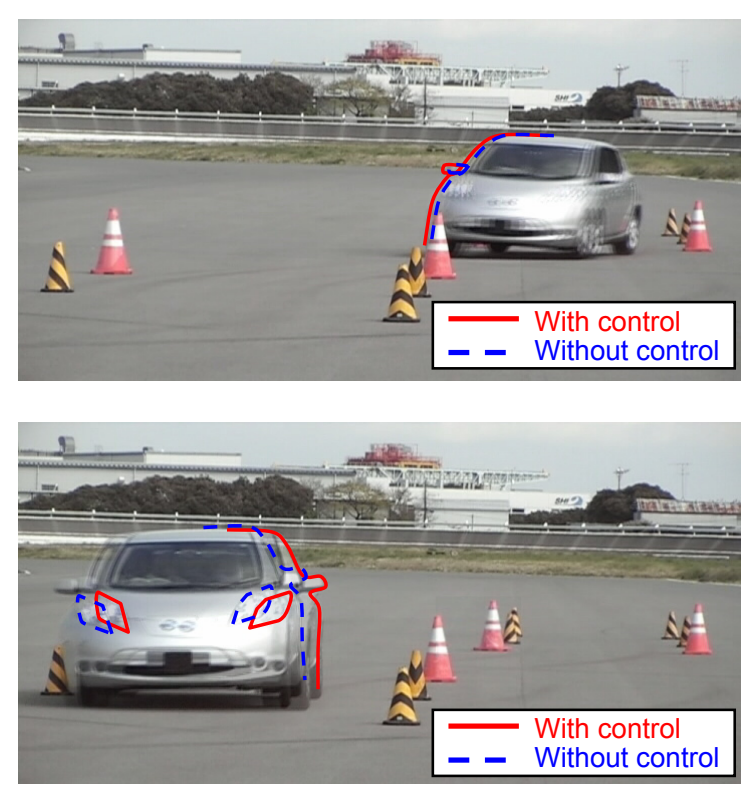

Figure 11: Effect of drive torque control

The photos in Fig. 11 indicate that increasing or decreasing the drive torque improved the initial yaw rate response, as seen in the vehicle behavior immediately after the start of the lane change. In addition, vehicle behavior in the lateral direction after crossing the lane is also seen to be improved, indicating that body lean behavior was suppressed.

The effect of the control system against the same force input was also confirmed on the basis of data measured with vehicle-mounted sensors. In this driving test, a steering input was applied as shown in Fig. 12 while the vehicle was traveling at a steady speed. Time histories of the yaw rate and roll rate measured at that time are shown in Figs. 13 and 14, respectively. The results in Fig. 13 show that the control system improved the initial yaw rate response to the steering input. Moreover, it is seen in Fig. 14 that the peak roll rate was suppressed compared with the result without the control system.

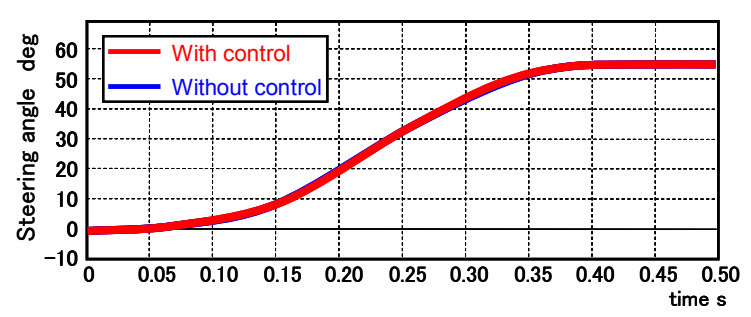

Figure12: Steering angle time history

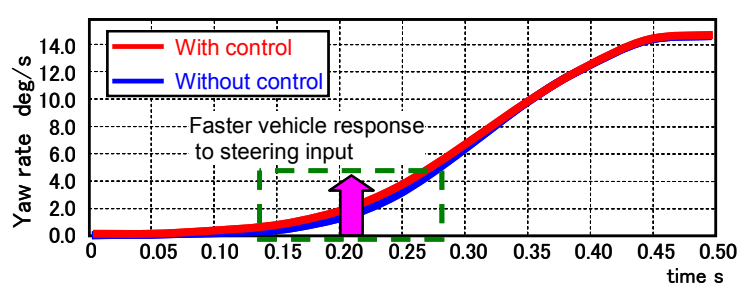

Figure13: Yaw rate time history

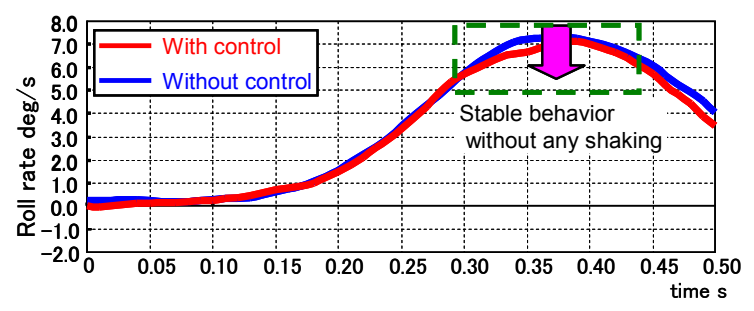

Figure14: Roll rate time history

\section{Conclusion}

Simulations and driving tests were conducted to validate the basic control principle and confirm the effects of drive torque control. The purpose was to verify whether the mechanism and control logic described above could achieve the nimble smoothness that was defined as the ideal driving performance of an EV. In the process of developing a new EV, the ideal driving performance desired of an EV was defined as nimble smoothness, and a mechanism was derived for achieving the targeted performance. Based on that mechanism, a new drive torque control system was developed for enhancing handling performance, as opposed to its conventional use for controlling longitudinal vehicle motion. The application of this control system to the newly developed $\mathrm{EV}$ has contributed to the attainment of the nimble, smooth driving ease that characterizes an EV. 


\section{References}

[1] KAWAMURA, HIROMICHI : HighlyResponsive Acceleration Control for the Nissan LEAF Electric Vehicle: SAE International, 2011-01-0397(2011)

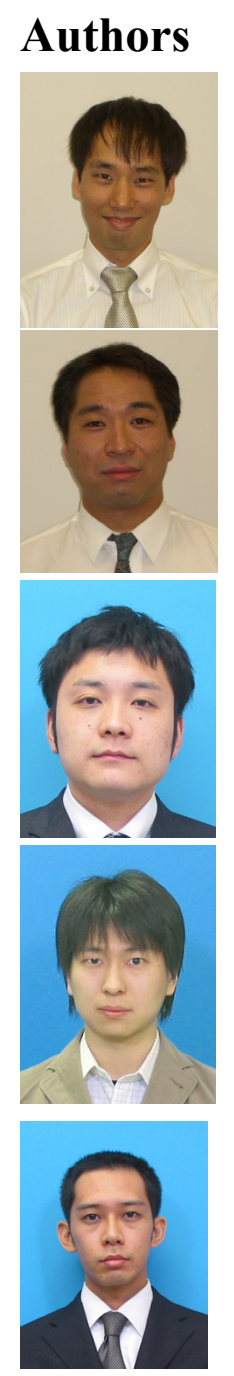

Yuuki Shiozawa

NISSAN MOTOR CO., LTD.

243-0123, 1-1 Morinosatoaoyama,

Atsugi-shi, Kanagawa, Japan

Tel:+81-50-2029-0323

E-mail: shioz@mail.nissan.co.jp

Yosuke Kobayashi

NISSAN MOTOR CO., LTD.

243-0123, 1-1 Morinosatoaoyama,

Atsugi-shi, Kanagawa, Japan

Tel:+81-50-2029-0834

E-mail: yo-koba@mail.nissan.co.jp

Mitsunori Ohta

NISSAN MOTOR CO., LTD.

243-0123, 1-1 Morinosatoaoyama,

Atsugi-shi, Kanagawa, Japan

Tel:+81-50-2029-0957

E-mail:

mitsunori_ohta@mail.nissan.co.jp

Toshiyuki Murata

NISSAN MOTOR CO., LTD.

243-0123, 1-1 Morinosatoaoyama,

Atsugi-shi, Kanagawa, Japan

Tel:+81-50-2029-0972

E-mail:

toshi-murata@mail.nissan.co.jp

Yusuke Kageyama

NISSAN MOTOR CO., LTD.

243-0123, 1-1 Morinosatoaoyama,

Atsugi-shi, Kanagawa, Japan

Tel:+81-50-2029-1320

E-mail:

yu-kageyama@mail.nissan.co.jp

Yohei Nakamura

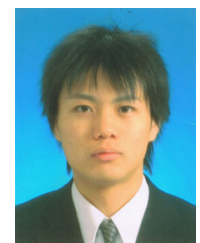

NISSAN MOTOR CO., LTD.

243-0192, 560-2, Okatsukoku, Atsugishi, Kanagawa, Japan

Tel:+81-50-2029-1757

E-mail:

yohei-nakamura@mail.nissan.co.jp

Hideaki Misawa

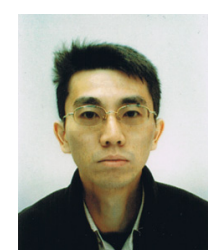

NISSAN MOTOR CO., LTD.

329-0692, $2500 \quad$ Kamigamou,

Kaminokawa-machi, Kawachi-gun,

Tochigi, Japan

Tel:+81- 285-56-1406

E-mail: h-misawa@mail.nissan.co.jp 